\title{
METHOD OF NON-CONTACT
}

MEASURING OF DIAMETER AND OVALITY OF STEEL TUBES DURING THEIR PRODUCTION

\section{DAVID FOJTIK, PETR PODESVA, JAN GEBAUER, JIRI CZEBE}

VSB - Technical University of Ostrava, Faculty of Mechanical Engineering, The Department of Control Systems and Instrumentation, Ostrava, Czech republic

DOI : 10.17973/MMSJ.2019_03_201898

Email: David.Fojtik@vsb.cz

The quality of production of metallurgical bar stock, in particular tubes, is assessed based on many production parameters including outer cross section (diameter) and ovality, which are also specified by European standard EN 13 508. Tubes need to be measured as soon as possible, ideally immediately after the rolling mill during their production, so that any defects may be corrected in due time and thus the loss of material and power may be minimized. This means that the tubes need to be measured at high temperatures (approximately $1.000{ }^{\circ} \mathrm{C}$ ). The article describes a new method of non-contact measuring of outer diameters and ovality of steel tubes cross sections that meets the above-mentioned requirements and allows reliable measuring of required parameters even in the case when the measured material moves or vibrates vertically during measuring.

KEYWORDS Laser scanning, 2D scanner, triangulation, diameter, ovality

\section{INTRODUCTION}

Outer diameters and ovality are crucial particularly in production of hot-rolled seamless steel tubes. Due to this production technology, diameter and ovality are monitored continually along the whole length of tubes; moreover, possible surface defects are detected, which may also indicate a defect of the rolling mill. It is also often required that these data are stored (at least throughout the warranty period) for the purposes of long-term production analysis or as a support means in case of claim procedures. In order to indicate correctly the cause of a defect, operators or inspectors need detailed data of the tube and a tool which would allow them a detailed analysis of the ascertained deformation.

Recently, the most common measurements have been carried out using contact, often manual, measuring instruments. The reason was either the low purchase cost or the lack of technology capable of performing these measurements. These methods have a number of drawbacks, the most significant of which are the impossibility of measuring objects moving at higher speeds or of objects with high surface temperatures.

Contact measurements can only be made after the object is at rest and its temperature drops. This creates the time gap between the origin of a defect in the manufacturing process and its detection. As long as the process defect is not detected, it will also affect other products and thus increase production costs. Requirements for precision in production and for lowering production costs are growing. Production waste forms large part of material, time and energy costs. The introduction of non-contact measurements into the production process is costly, especially due to difficult conditions of the measurement, but helps to minimize the possible damage. Great advantage of measurement using 2D or 3D scanners when comparing to other methods is that these scanners allow to acquire more values [Vijay 2017, Zbozinek 2017]. The scanners can detect defects that would otherwise be hidden and would result in returns made by customers and thus cause great damage to property and reputation of the manufacturer.

\section{SEAMLESS TUBES ROLLING MILL}

One of the production areas where these measurements are required is rolling of tubes (Fig. 1). The dimensions obtained serve as part of the output control as well as for the detection of a defect of the rolling mill system, especially the wear of the rollers. The measurement system takes place at the exit of the rolling mill at a temperature of the tubes in the range of 800 to $1000^{\circ} \mathrm{C}$ beside the water fog cooling chamber. Combining high temperature, water fog and ubiquitous dust creates a very hostile environment for the measuring system.

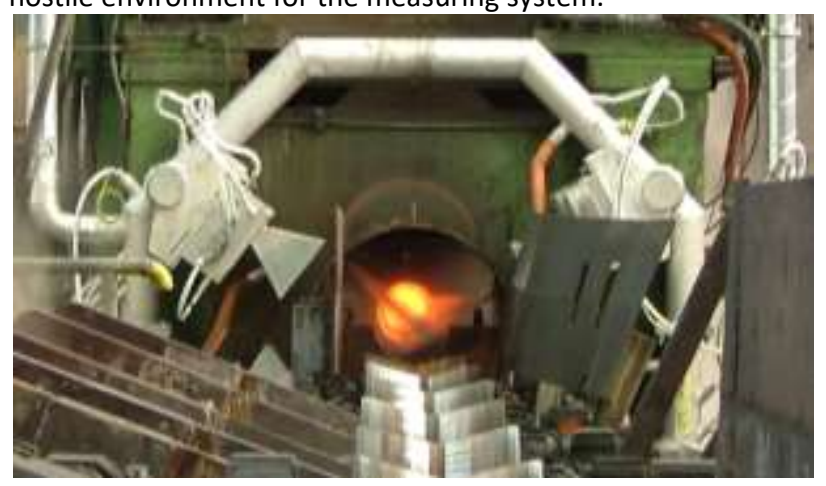

Figure 1. Rolling mill with scanning system

Rolling mill (Fig. 2) is used to correct proportions of semi rolled seamless pipes to the required size. It consists of a furnace in which the semi-finished product is heated to the desired temperature and is formed to the desired dimension by several sets of rollers with graduated radial. Before leaving of the rolling mill, the product is cooled by water mist and then nested on the conveyor. The end of the blank on the output of the mill is supported to avoid deformation of blank and suppress the vibration of the free end.

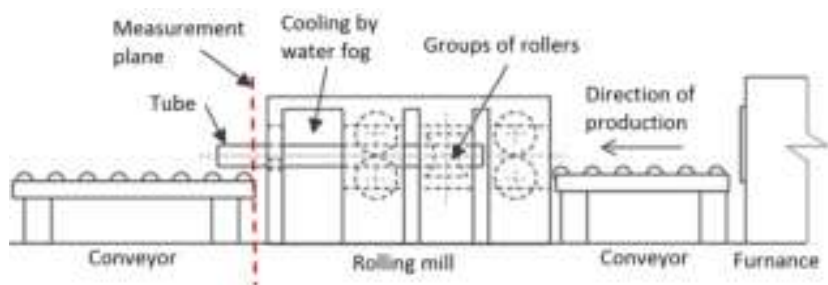

Figure 2. Scheme of the rolling mill

The calibrated tube is then transported to the cooling storage where it's cooled by air or by water fog to the handling temperature. Cooling will reduce the dimensions caused by thermal expansion of the material. The change in dimension against the measured value is corrected using conversion tables. The rolled pipe is subsequently machined to exact dimensions in another workplace.

\section{SCANNING SYSTEM}

This system is based on a group of synchronized 2D triangulation scanners located in one plane around a common center (Fig. 3). Scanners are positioned on the frame serving to 
distribute the necessary refrigerants and signal and power conductors. During scanning, they receive information about the distance from individual points on the surface of the tube, and these data are then recalculated, refined and processed by a computer equipped with a suitable interface and, in particular, the analysis software. Using a special algorithm, the software determines the diameter and ovality over the entire length of the tube that is measured. Information is displayed on a remote workstation and stored in a database.

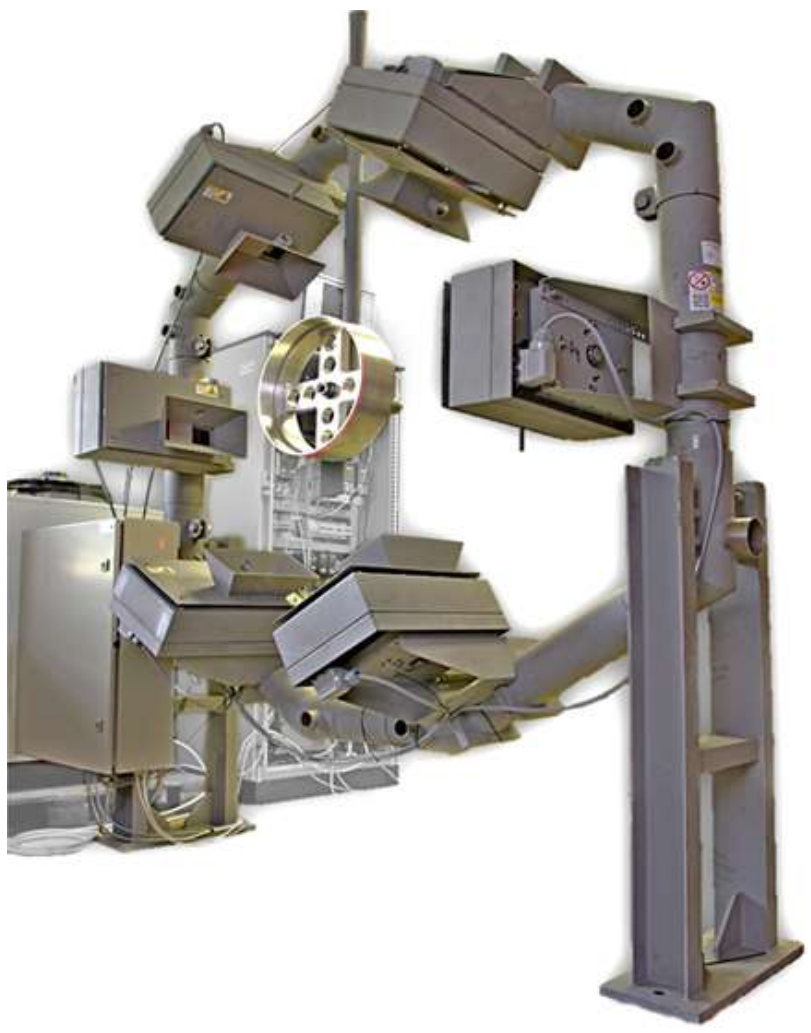

Figure 3. Modular system for scanning

\subsection{Why triangulation floating point scanner}

2D scanners can be classified by two aspects, by method of distance measurement and by method of obtaining the line of scan.

Distance measurement can be based on time of flight of the light from transmitter to target and back to receiver (TOF). This allows range from few tens of $\mathrm{mm}$ to tens of $\mathrm{km}$ (in exceptional cases hundreds of thousands of kilometres [Chapront 2006]). Range capability is redeemed by lower resolution and accuracy that depends on frequency and precision of internal clock. It can't reach level of other methods like these based on triangulation or phase measurement. Triangulation method is based on determining the position of a reflected point on the linear light sensor. This sensor observes whole measurement range at a certain distance from laser source. This type of sensors has very limited range and work distance, from few $\mathrm{mm}$ to around $1 \mathrm{~m}$, but accuracy and resolution is usually between $0,05 \%$ and $0,005 \%$ of range.

Line of scan can be created from spot beam by shaping lens to homogenous line or by mechanical movement of the spot beam, so called flying spot. Advantage of homogenous line is absence of moving part so system can be simpler, smaller and more reliable. And mainly, the whole line is created at the same time. The shape of obtained image is not affected by movement of scanned object during measurement as in case of flying spot method as shown on fig. 4. But there are a few disadvantages of this solution. Laser source for shaped line must be much more powerful than in the case of flying spot, where all energy is concentrated in small area. Angle of view is limited because it's more difficult to shape beam in wide angle with same sharpness and intensity on whole area. Similar problem is with acquisition of image because wide angle lenses cause distortion of image. Another problem is caused by differences in reflexivity of material along the projected line. Even when surface reflectivity changes, it is more reliable to get pivot of single spot than of a single line where nearby points are blended together and their shape and size vary by reflexivity of surface.

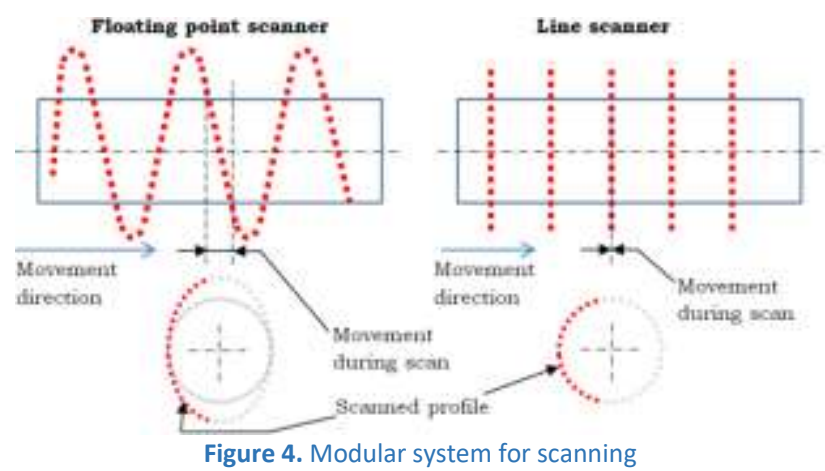

\subsection{Synchronization of scanners}

If the scanners are placed in one plane and run independently, there will be situations (Fig. 5) where one scanner sees the point created by the second scan beam. This will cause the sensor to detect point mistakes and incorrectly determine the distance. It is therefore necessary to synchronize the movement of the beams of all scanners. Solution is synchronization of the movement of all scanners, so they all keep same angle at any time.

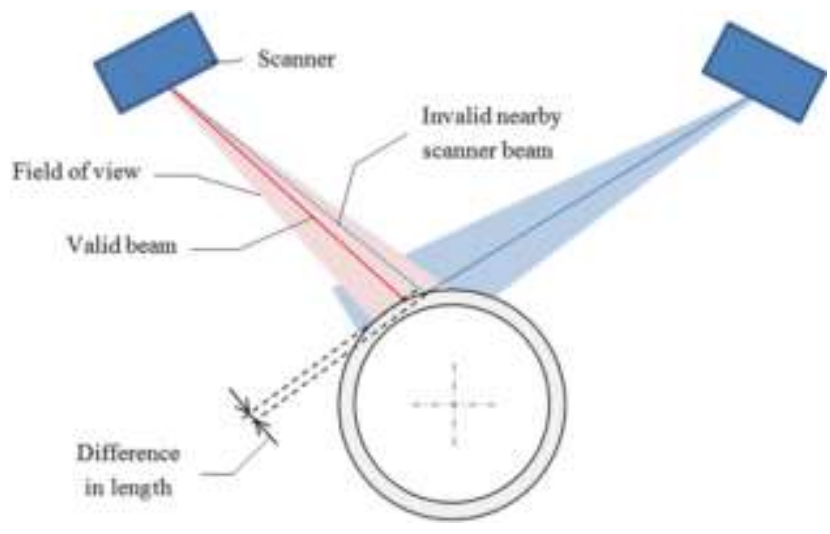

Figure 5. Effect of non-synchronized scanners

The synchronization is also needed to ensure that samples from all scanners are acquired at the same time; otherwise phase shifts may occur. In the case of a cylindrical body, it would be reflected at a misdirected point in relation to other points.

\section{APPLICATION TUBESBROWSER}

Application TubesBrowser (Fig. 6) is used to visualize the stored data representing the set of surface points measured above hot-formed steel tubes. The program provides the ability to view sub-sections of each tube scan and calculates the maximum, minimum and overall tube diameter and ovality from these scans. This information is displayed in a graph representing the variation of these values across the entire length of the tube, and also in text boxes for each profile. During measurement, it is necessary to use two filters which together serve to eliminate erroneously measured points. 


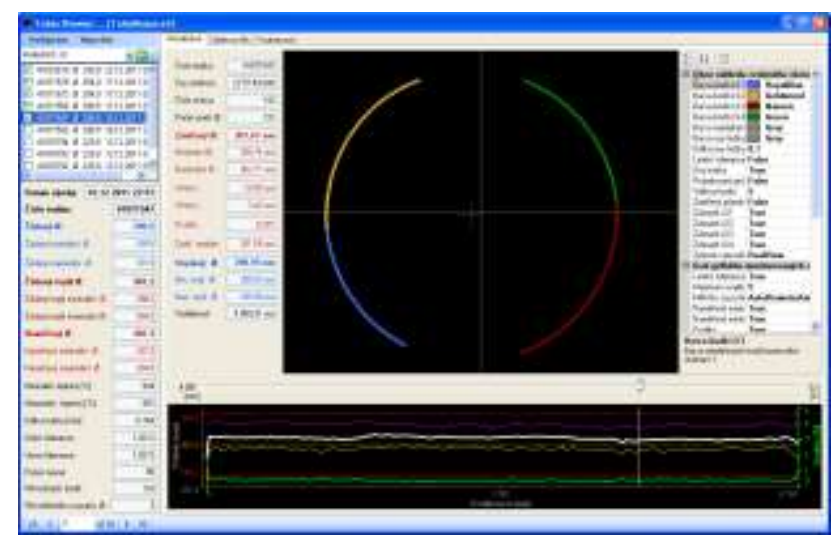

Figure 6. Tube Browser - database viewer

\subsection{Filtering of tangential points}

This filter (Fig. 7) is used separately in individual coordinate systems of scanners. The only points used are those for which $|\Delta x / \Delta y|<F T B$ applies, where $\Delta x$ and $\Delta y$ are distances between two consecutively measured points of one scan in the $x$ and $y$ axes. In essence, this determines the angle of covering the tube with one scanner. It is advisable to use filters with values of 0.8 and lower (in this case, each scanner covers about $78^{\circ}$ of the tube segment) whereas the valid values of the filter are in the range of $0.1 \div 2.5$ (coverage angle $12^{\circ} \div 136^{\circ}$ ).

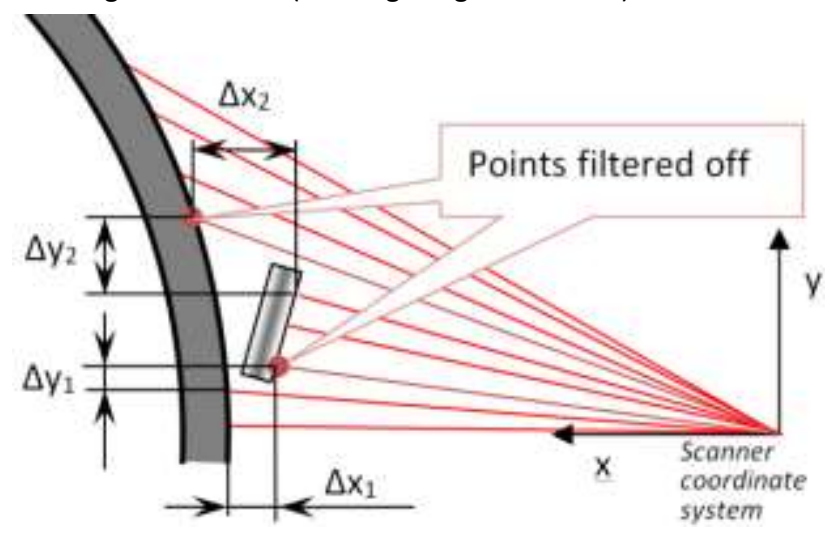

Figure 7. Tangential filter

Mathematically, $\Delta x / \Delta y$ expresses the slope of the bisector line passing through two adjacent points. For very close points $(\Delta x / \Delta y \rightarrow 0)$, this bisector line passes into a tangent line that can be analytically expressed by derivative of the circle function $\sin (\alpha) / \cos (\alpha)=\operatorname{tg}(\alpha)=$ FTB. For the desired maximum angle of tube coverage by the scanner, $\beta=2 \cdot \alpha$, it is then easy to determine the value of the filter. For a complete coverage of the tube by four scanners rotated by $90^{\circ}$ (coverage angle $\beta=$ $90^{\circ}$ ), the angle $\alpha=45^{\circ}$. Therefore, the filtration coefficient is $\operatorname{tg}\left(45^{\circ}\right)=1$. In addition to filtration of points located outside the coverage angle, the filter also eliminates erroneously measured solitary points.

\subsection{Filter of the measured range of diameters:}

This filter eliminates the measured diameters whose values are outside the specified range which is defined as a multiple of manufacturing tolerances of the tube. The value of the filter is a positive number in the interval of $1 \div 25$ which determines the valid interval after multiplying the upper and lower manufacturing tolerances.

For example, for a filter with the value of 3, rolled tube with diameter of $194 \mathrm{~mm}$, upper manufacturing tolerance of $1 \%$ and lower manufacturing tolerance of $0.5 \%$, the valid interval is 191 $\div 199.8(194-194 \cdot 0.005 \cdot 3 \div 300+194 \cdot 0.01 \cdot 3)$. Any measured diameter outside this range is ignored; subsequently, the points outside the measured interval are analyzed and then marked as invalid. In the TubeBrowser application, the invalid points are implicitly shown in gray (Fig. 8).
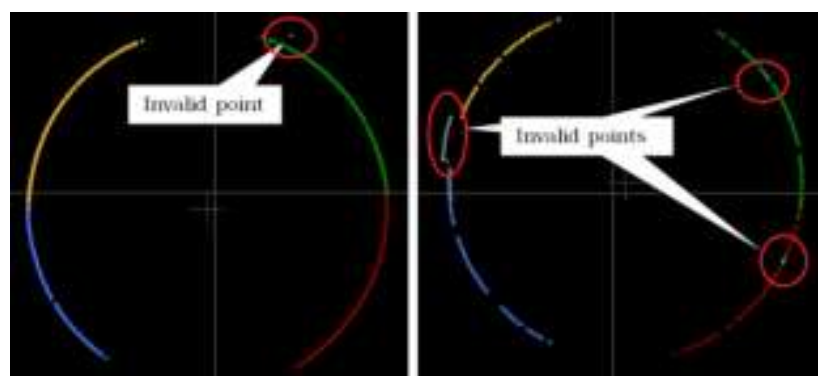

Figure 8. Example of invalid points filtration

The diameter is always measured from three simultaneously measured points. Four-scanner measurements often provide four points measured simultaneously. Then, in total four diameters are calculated (4! / 3!). To determine invalid points (points that are not used for the diameter calculation), the application uses an algorithm that first determines the center of the tube and then, based on the point distance from the tube center, marks those whose distance is beyond the halfinterval of the measured diameter range.

\subsection{Database storage TubeMeasured}

Basic repository for tube measurement data consists of a database SQL in server 2008 R2 that is popular as storage method [Zhang 2017] together with XML database [Smutný 2011]. The database is designed to minimize demands on storage space while maintaining rapid system response in data retrieval. As the number of points for each measured tube is very high, the table is designed to maximally save space even with an acceptable loss of accuracy. [Fojtik 2015] Coordinates of points in millimeters are stored as 16-bit integers so that the original real coordinate values are rounded to one decimal place and multiplied by ten. This provides an integer which stores coordinates with an accuracy of one tenth of millimeter in the range of about $\pm 3200 \mathrm{~mm}$, which is a sufficient range (in reality, it moves up to $\pm 800 \mathrm{~mm}$ ), while maintaining sufficient accuracy that is higher than the accuracy of measurement scanners alone. In case of more accurate scanners, after adjustments (the value would be multiplied by 20 or maximally by 40 ), it would be possible to reach higher accuracy ( 0.05 or maximally 0.025 ) at the expense of a reduction in range $( \pm 1600, \pm 800$ ). With regard to the measurement accuracy provided by scanners, however, this is not needed.

\section{ALIGNMENT OF SYSTEM}

It is clear that the positioning of individual scanners will also have a major influence on the accuracy and linearity of the measurement. After installation of the system, scanners are not aligned correctly. Any deviation of the scanner position from the work plane will result in a distortion of the acquired image. The same way is represented by the distortion of the image and the inclination of the body to the working plane. This also applies to calibration products designed to adjust the system. Tilting and moving the body toward the scanner's working plane has a clear effect on the shape of the image obtained. An impact analysis has been carried out to determine tolerance for deviations to be achieved in order not to impair the required accuracy of the measuring system. Influences are as follows: 


\subsection{Influence of the shift}

It can be seen from fig. 9 that the displacement affects the coverage of the circular profile, the resolution and the angle of impact of the beam on the surface. With the increasing distance, the resolution of the scanned area decreases as the adjacent points fall on the surface of the larger spacing. The analysis also showed that the displacement in the $y$ axis has a significant effect on the resulting measurement deviation.

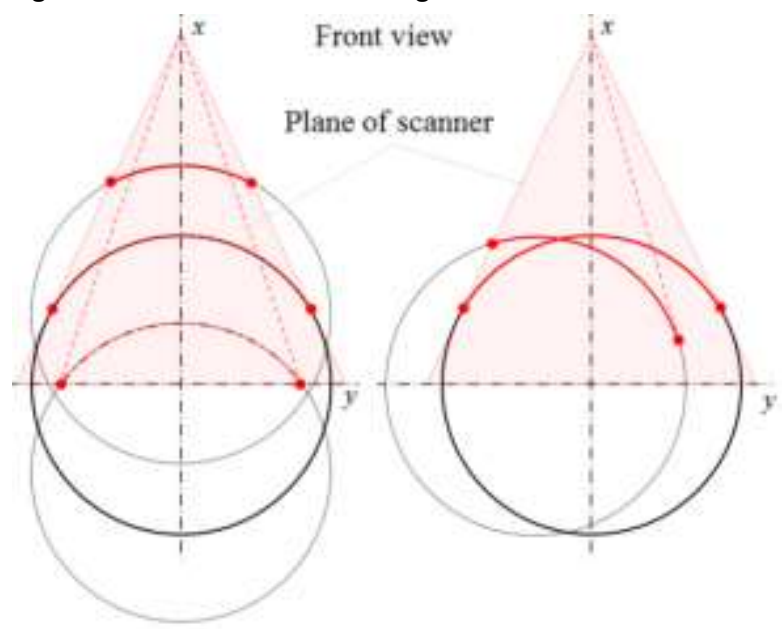

Figure 9. Influence of the shift on scan in $\mathrm{x}$ and $\mathrm{y}$ axes

\subsection{Influence of the tilt}

By tilting the body with a circular cross-section to the plane of the scanner, we obtain an ellipse-shaped image, as shown in fig, 10. If it is not known that the tilt occurs, the image will be distorted by movement of the points thus resulting in incorrect calculation of diameter and the ovality of the body. If the angle of tilt in all axes is known, a geometric correction can be made. In practice, measuring these angles is very difficult.

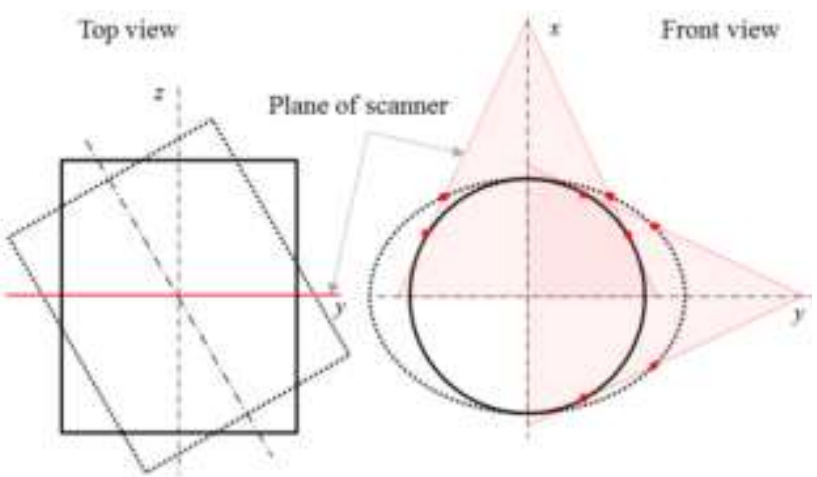

Figure 10. Influence of the tilt on scan in $\mathrm{x}$ and $\mathrm{y}$ axes

\subsection{Analysis of two-axis tilt and shift}

For simulation of image of cylinder affected by tilt and shift distortion, we have to calculate position of the contact point of the scanner beam with the surface of the cylindrical body. Solution consists of the part in the z-plane that is perpendicular to the axis of the measured body and the part with conversion between this plane and the work plane of the scanner. $\alpha z$ is the beam angle from the calibration $\mathrm{DLL}$, the $\mathrm{Hz}$ and az determines scanner workspace in requested half-plane, xo and yo are offsets of the circle and $r$ its radius. Equations 11 and 12 determine the coordinates of the desired point in the $z$-plane.

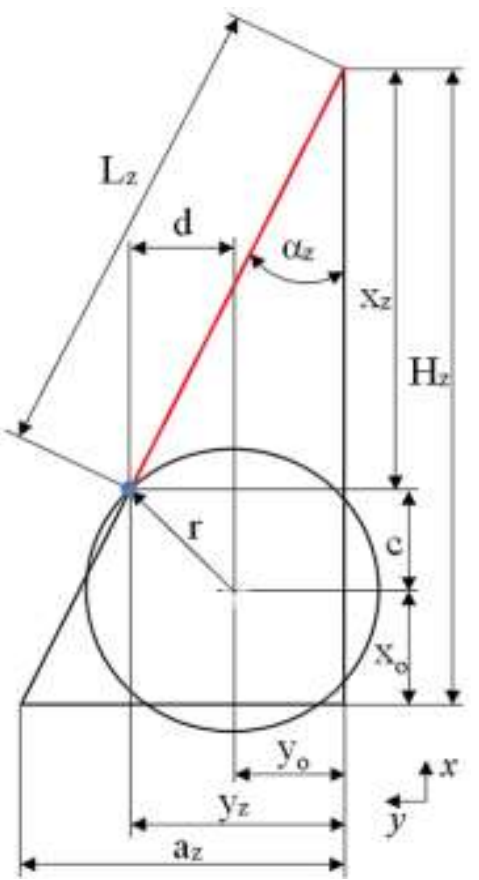

Figure 11. Analysis of impact on tilted and shifted round object

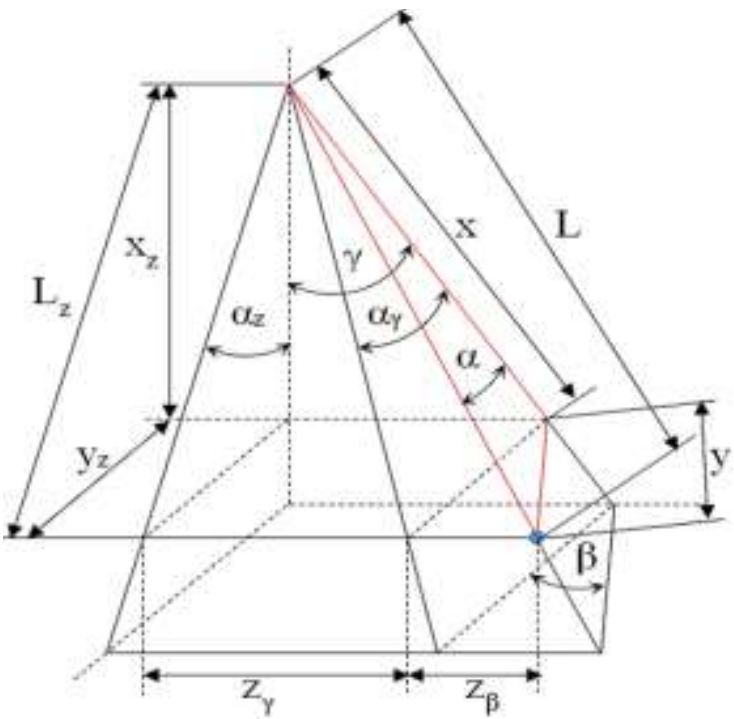

Figure 12. Analysis of impact on tilted and shifted round object

The coordinates of the point at the inclination of the plane of the scanner according to the $y$-axis and its rotation according to the z-axis (Fig. 12) is based on equations 1-9, the projected beam angle $\alpha z$ being given by equation 10 .

$\frac{d+y_{0}}{H_{z}-x_{0}-c}=\frac{a_{z}}{H_{z}}$

$d^{2}=r^{2}+c^{2}$

$A c^{2}+B c+C=0$

$A=\frac{a_{z}^{2}}{\left(H_{z}-x_{0}\right)^{2}}+1$

$B=\frac{2 a_{z}}{H_{z}-x_{0}}\left(y_{0}-a\right)$

$C=a_{z}^{2}-2 a_{z}^{2} y_{0}+y_{0}^{2}-r^{2}$

$c=\frac{-B+\sqrt{B^{2}-4 A C}}{2 A}$

$x_{z}=H_{z}-x_{0}-c$

$y_{z}=x_{z} \cdot \operatorname{tg}\left(\alpha_{z}\right)$

The recalculation of projected coordinates back to the work plane of the scanner (fig. 11) is given by equations 11 and 12 . 


$$
\begin{aligned}
& \alpha_{z}=\tan ^{-1} \frac{\cos \beta \tan \alpha}{\cos \gamma} \\
& x=\frac{x_{z}}{\cos \gamma} \\
& y=\frac{y_{z}}{\cos \beta}
\end{aligned}
$$

\subsection{Scanning system adjustment}

According to previous analysis, method was developed to adjust group of scanners into single working plane by the set of gauges and reference laser beam (Fig. 13). There are many ways to calibrate scanners [Meng 2015] In this method, the beam was set parallel to the axis of the mill. The axis of measurement system should be as close and parallel to axis of mill as possible. Next, hollow carrier mandrel of the gauge was placed so that the beam passes through the aperture in the mandrel. A rotatable gauge with sliding ruler was used with a sliding ruler and gradually set all scanners to the plane defined by the edge of the ruler while rotating the pattern (fig. 14). This means that the line formed by the scanner beam touches the edge of the ruler when rotating the pattern. According to simulations, this allows us to decrease error in angle below $1^{\circ}$ and measurement error caused by tilting below $0.1 \mathrm{~mm}$ on tube with $500 \mathrm{~mm}$ diameter.

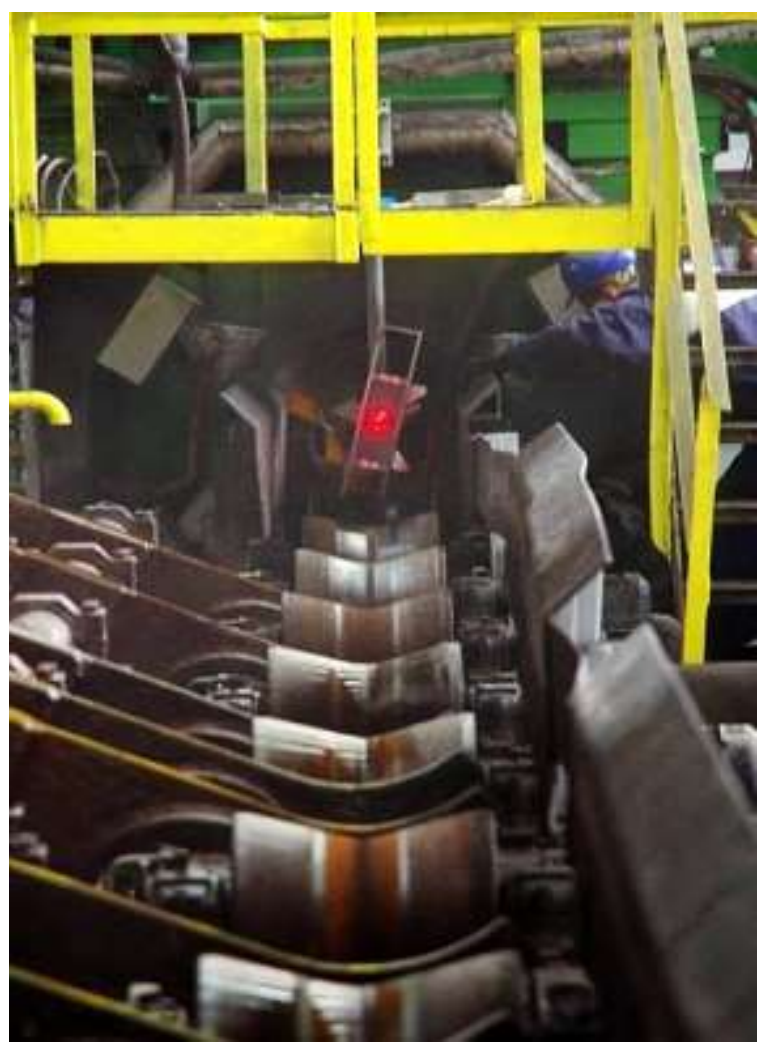

Figure 13. Scanner adjustment with gauge

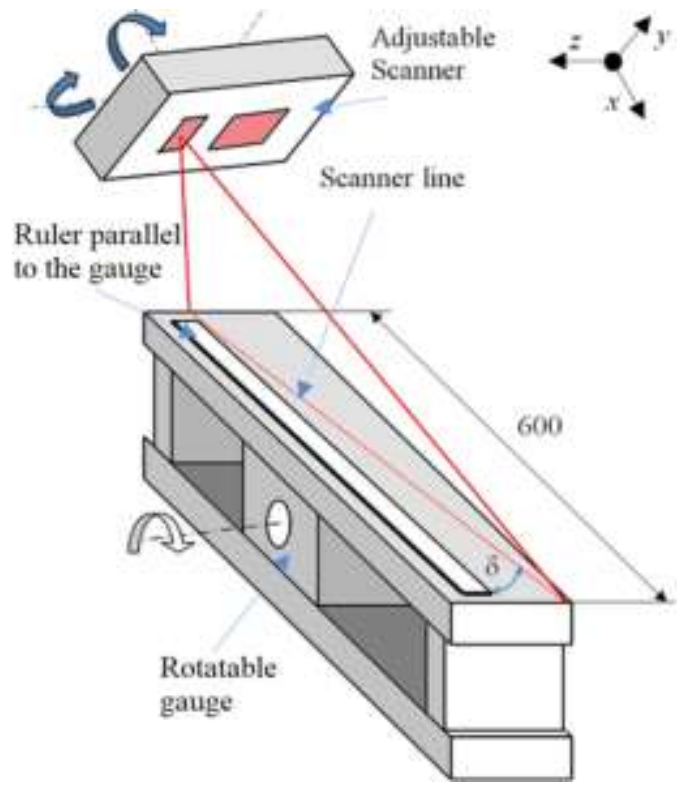

Figure 14. Scanner adjustment with gauge

\section{CONCLUSION}

The number of laser scanner applications in the industry is still growing due to lower prices, improved performance and growing industry interest in lowering production costs by quality control during production. Laser rangefinders, 2D and $3 D$ scanners have unique features that other types of scanners are not able to offer. This project was focused on use of $2 \mathrm{D}$ laser scanners for measurement of diameter and ovality of pipes at the exit from the rolling mill. New measurement system capable of operating at high temperatures and pipe movement was successfully implemented in industry.

This project also focused on improvement of quality of scanner systems used in tube diameter and ovality measurements and the adjustment of its sensors. The method serves as a preparatory step for software scanner calibration and creation of a common coordinate system. Thanks to a much more precise adjustment of the scanners to one working plane, which was also perpendicular to the center line of the rolled pipe with an estimated deviation of up to $1^{\circ}$, we achieved a total scatter value of $\pm 0.5 \mathrm{~mm}$ for the measured diameters and ovality.

\section{ACKNOWLEDGMENTS}

This work was supported by the European Regional Development Fund in the Research Centre of Advanced Mechatronic Systems project, CZ.02.1.01/0.0/0.0/16_019/0000867 within the Operational Programme Research, Development and Education and the project SP2019/51 Applied Research in the Area of Machine and Process Control supported by the Ministry of Education, Youth and Sports.

\section{REFERENCES}

[Chapront 2006] Chapront, J. and Francou G. Lunar Laser Ranging: measurements, analysis, and contribution to the reference systems. IERS Tech. Note, 34 (2006), pp. 97-116

[Fojtik 2015] Fojtik, D., et al. Storing high volumes of data in MS $\mathrm{SQL}$ server express. Paper presented at the Proceedings of the 2015 16th International Carpathian Control Conference, ICCC 2015, 125128. doi:10.1109/CarpathianCC.2015.7145059 
[Łuczak 2017] Łuczak, S., et al.. Selection of MEMS Accelerometers for Tilt Measurements (2017) Journal of Sensors, 2017, art. no. 9796146, DOI: $10.1155 / 2017 / 9796146$

[Meng 2015] Meng, Q. X., et al. Calibration method of the 3-D laser sensor measurement system. In: Yang C., Virk G., Yang H. (eds) Wearable Sensors and Robots. Lecture Notes in Electrical Engineering, vol 399. Springer, Singapore, ISBN: 978-981-10-2404-7

[Smutny 2011] Smutny, P., Visual programming for smartphones. 12th International Carpathian Control Conference (ICCC), Velke Karlovice, 2011, pp. 358361. doi: 10.1109/CarpathianCC.2011.5945879

[Vijay 2017] Vijay, A., et al. Development of an economical digital control method for a continuously running conveyor belt. International Conference on Inventive Systems and Control (ICISC), Coimbatore, 2017, pp. 1-5. doi: 10.1109/ICISC.2017.8068619
[Zbozinek 2017] Zbozinek, V., et al. Car axle testing on hydropulse. 8th International Carpathian Control Conference (ICCC), Sinaia, 2017, pp. 1-4. doi: 10.1109/CarpathianCC.2017.7970360

[Zhang 2017] Zhang, Y., et al. The intelligent parking lot based on ZigBee technology and SVM. 14th IEEE Annual Consumer Communications \& Networking Conference (CCNC), Las Vegas, NV, 2017, pp. 620621. doi: 10.1109/CCNC.2017.7983194

\section{CONTACTS:}

Ing. David Fojtik Ph.D

VSB - Technical University of Ostrava, Faculty of Mechanical Engineering, The Department of Control Systems and Instrumentation

17. listopadu 2172/15, Ostrava, 70800, Czech republic +420 597324 193, david.fojtik@vsb.cz, http://www.352.vsb.cz/ 